\title{
Numerical Simulation and Experiment of Hydraulic Loss of Two-Stage Flap Valves
}

\author{
Bingnan ZHOU, Weigang LU ${ }^{1}$, Bo XU and Hua CHEN \\ College of Hydraulic Science and Engineering, Yangzhou University, Yangzhou, \\ Jiangsu, China
}

\begin{abstract}
As a type of flap valve evolved from integral flap valve, two-stage flap valve has the advantages of large opening angle, small hydraulic loss and small impact force on the flap valve seat when the flap valve is closed. In order to analyze and study the hydraulic loss characteristics of the two-stage flap valve, this paper takes a pump station as an example. Based on theoretical analysis, combined with numerical simulation and model test, the hydraulic loss of two-stage flap valve is studied, and the relationship between hydraulic loss and pump station flow is obtained. According to the test results, the hydraulic loss of two-stage flap valve increases with the increase of flow rate under the same opening angle of flap valve. Under the same flow condition, the larger the opening angle of the flap valve is, the smaller the hydraulic loss of the two-stage flap valve is. When the opening angle of the upper flap valve is greater than $46^{\circ}$ and the opening angle of the lower flap valve is greater than $64^{\circ}$, the hydraulic loss is less than $70 \mathrm{~mm}$ and tends to be stable. The influence of hydraulic loss on the performance of pump device is gradually weakened. The relationship between hydraulic loss and flow of two-stage flap valve no longer satisfies the relationship of square under the constant opening angle. Moreover, the larger the opening angle of the two-stage flap valve is, the greater the relationship between hydraulic loss and flow is. Compared with the integral flap valve, the two-stage flap valve has better structural form and hydraulic characteristics, and has little influence on the performance of the pump device, which can provide reference for the application of the two-stage flap valve in the pump station.
\end{abstract}

Keywords. Two-stage flap valves, hydraulic loss, pump station

\section{Introduction}

The opening angle and hydraulic loss of the flap valves, as a measure to cut off the flow in the pumping station, are more concerned by scholars ${ }^{[1]}$. To solve these problems, on the basis of the traditional flap valves, people have carried out some transformation, and there are abundant flap valve types. The two-stage flap valves are a kind of flap valve type evolved from the integral flap valve. Compared with the integral flap valve, the two-stage flap valves have the advantages of large opening angle, small hydraulic loss and small impact force on the gate seat when the flap valve is closed. Scholars have carried out research on the integral flap valve and the two-stage flap valves. Zhu et al. [1] studied the cause of head loss of two-stage flap valves. Yang et al. [2] explored the hydraulic loss of integral flap valve by model test combined with

${ }^{1}$ Corresponding Author, Weigang LU, College of Hydraulic Science and Engineering, Yangzhou University, Yangzhou, Jiangsu, China; Email: wglu@yzu.edu.cn. 
numerical simulation. Zhu et al. [3] conducted an in-depth study on the causes of hydraulic loss of integral flap valve and the influence of hydraulic loss of flap valve on pump efficiency. He et al. [4] studied the relationship between hydraulic loss and discharge area of integral flap valve. Chu et al. [5, 6] discussed the opening process, opening angle empirical formula and its movement in still water, and carried out numerical simulation and model test research on its opening angle. Generally speaking, there is little research on the hydraulic characteristics of the two-stage flap valves, which needs to be further studied.

Aiming at the problem of hydraulic loss of two-stage flap valves, this paper takes the two-stage flap valves of a pumping station as an example. Based on the theoretical analysis, combined with numerical simulation and model test, the hydraulic loss of the two-stage flap valves is studied. Through the research, the hydraulic loss characteristics of the two-stage flap valves and the relationship between the hydraulic loss and the flow of the pump station are obtained. It provides a reference for the popularization and application of two-stage flap valves in pumping station.

\section{Brief Introduction of Examples}

Taking the two-stage flap valves of a drainage pumping station as the research object. Four tubular pumps with $\mathrm{D}=2.25 \mathrm{~m}$ are selected for the pumping station. The design head is $2.28 \mathrm{~m}$, the maximum net head is $3.75 \mathrm{~m}$, the average net head is $0.4 \mathrm{~m}$, the minimum net head is $0.3 \mathrm{~m}$, the supporting unit capacity is $850 \mathrm{~kW}$, and the design flow of single pump is $16 \mathrm{~m}^{3} / \mathrm{s}$. The rectangular two-stage flap valves are selected. The flap valve is made into a plane steel plate structure. The width of the flap valve is $b=$ $3.24 \mathrm{~m}$, the total height is $\mathrm{h}=3.30 \mathrm{~m}$, and the hinge section of the lower gate is $0.4 \mathrm{~m}$ between the upper and lower gates. The design height of the upper gate is $1.7 \mathrm{~m}$ and that of the lower gate is $1.2 \mathrm{~m}$. In the static state, the inclination of the flap valves are shown as the angle between the center line of the runner at the outlet of the pump station and the horizontal plane. The angle is $\alpha_{\mathrm{m}}=10^{\circ}$.

\section{Theoretical Analysis}

\subsection{Style and Spacing}

The opening angle of the two-stage flap valves of the pump station is calculated by the equation in Ref. [6]:

$$
\left\{\begin{array}{l}
\frac{M_{G 2}}{M_{P 2}}=\frac{\cos ^{2} \phi_{2}}{\sin \phi_{2}\left(1-\cos \phi_{2}\right)} \\
\frac{M_{G 1}}{M_{P 1} \cos \Delta \phi}=\frac{\cos ^{2} \phi_{1}}{\sin \phi_{1}\left(1-\frac{h_{1}}{h} \cos \phi_{1}\right)}
\end{array}\right.
$$

where: $h$ is the total height of the two-stage flap valves; $h_{1}$ is the height of the upper beat gate; $\phi_{1}, \phi_{2}$ is the opening angle of the upper and lower gates of the two-stage flap valves; $\Delta \phi$ is the opening angle difference between the upper and lower gate; $M_{\mathrm{G} 1}, M_{\mathrm{G} 2}$ 
is the buoyancy moment of upper and lower gate; $M_{\mathrm{P} 1}, M_{\mathrm{P} 2}$ is the flow impulse moment of upper and lower throttle respectively.

In order to study the opening angle of the two-stage flap valves in the process of operation, the opening angle of the upper and lower flap valve when they can maintain torque balance is mainly calculated under the flow condition of $2 \mathrm{~m}^{3} / \mathrm{s} \sim 16 \mathrm{~m}^{3} / \mathrm{s}$. The calculation results of the opening angle of the two-stage flap valves are shown in table 1 .

\subsection{Theoretical Calculation Method of Hydraulic Loss}

The hydraulic loss coefficient of two-stage flap valves is calculated by using the empirical formula derived by Zhu [1]:

$$
\left\{\begin{array}{l}
\xi_{p}=1305.7 e^{A h_{1}+B \phi_{1}+C \phi_{2}} \\
A=6.02625-8.7349 \frac{h_{1}}{h}+4.2180\left(\frac{h_{1}}{h}\right)^{2}-0.09205 \phi_{1}+0.05183 \phi_{2} \\
B=0.04974-5.334 \times 10^{-5} \phi_{1}-8.84 \times 10^{-4} \phi_{2} \\
C=-0.2544+2.975 \times 10^{-3} \phi_{2}-1.369 \times 10^{-5} \phi_{2}^{2}+5.614 \times 10^{-4} \frac{h_{1}}{h} \phi_{1}
\end{array}\right.
$$

Table 1. The results of the opening angle of each scheme.

\begin{tabular}{lll}
\hline Flow scheme $\left(\mathbf{m}^{\mathbf{3}} \cdot \mathbf{s}^{-\mathbf{1}}\right)$ & Angle of upper gate $\left(^{\circ}\right)$ & Angle of lower gate $\left(^{\circ}\right)$ \\
\hline 2 & 14.02 & 31.98 \\
4 & 24.99 & 47.13 \\
6 & 32.49 & 54.36 \\
8 & 38.22 & 58.67 \\
10 & 42.34 & 61.61 \\
12 & 45.84 & 63.81 \\
\hline 14 & 48.76 & 65.65 \\
16 & 52.25 & 66.99 \\
\hline
\end{tabular}

Theoretical hydraulic loss of two-stage flap valves $\Delta \mathrm{h}_{\mathrm{p}}$ is calculated by the following formula:

$$
\Delta h_{p}=\frac{\xi_{p} v^{2}}{2 g}
$$

where: $v$ is the flow rate at the pump outlet; $g$ is the acceleration of gravity; $\xi_{\mathrm{p}}$ is the head loss coefficient of flap valve.

\subsection{Theoretical Calculation Results of Hydraulic Loss}

Taking the data into equations (2) and (3), the theoretical hydraulic loss of flap valve under each scheme can be obtained. The results are shown in table 2. 
Table 2. Hydraulic loss of flap gate with different flow rates.

\begin{tabular}{lllll}
\hline $\begin{array}{l}\text { Flow scheme } \\
\left(\mathbf{m}^{\mathbf{3}} \cdot \mathbf{s}^{-\mathbf{1}}\right)\end{array}$ & $\begin{array}{l}\text { Upper gate } \\
\text { opening angle }\left({ }^{\circ}\right)\end{array}$ & $\begin{array}{l}\text { Lower gate } \\
\text { opening angle }\left(^{\circ}\right)\end{array}$ & $\begin{array}{l}\text { Loss } \\
\text { coefficient }\end{array}$ & $\begin{array}{l}\text { Local hydraulic loss of } \\
\text { flap valve }(\mathbf{m m})\end{array}$ \\
\hline 2 & 14.02 & 31.98 & 2.197 & 254.97 \\
4 & 24.99 & 47.13 & 0.837 & 122.08 \\
6 & 32.49 & 54.36 & 0.477 & 92.22 \\
8 & 38.22 & 58.67 & 0.323 & 79.97 \\
10 & 42.34 & 61.61 & 0.245 & 76.78 \\
12 & 45.84 & 63.81 & 0.196 & 75.17 \\
14 & 48.76 & 65.65 & 0.162 & 74.42 \\
16 & 52.25 & 66.99 & 0.139 & 75.36 \\
\hline
\end{tabular}

According to the data in the analysis table, with the increase of the flow, the twostage flap valves are gradually opened, and the opening angle of the lower beat gate is larger than that of the upper beat gate. During the opening process of the two-stage flap valves, the water section increases gradually, the hydraulic loss coefficient of the flap valve decreases gradually, and the hydraulic loss also decreases gradually. When the discharge increases to $12 \mathrm{~m}^{3} / \mathrm{s}$, the hydraulic loss of outlet section tends to be stable, and the size is only about $75 \mathrm{~mm}$. At this time, the opening angle of the upper beat gate is greater than $45.84^{\circ}$, the opening angle of lower beat gate is greater than $63.81^{\circ}$. Compared with the hydraulic loss coefficient of the integral flap valve in Ref. [7] in the same flow range and the opening angle of the flap valve is $45^{\circ}$, the hydraulic loss coefficient of the two-stage flap valves is smaller than that of the integral flap valve. It shows that the two-stage flap valves have a better structure than the integral flap valve.

\section{Numerical Simulation}

\subsection{Model Building}

According to the engineering design drawings of the pumping station and related data, use NX 11.0 to model the calculation area at the exit of the pumping station. The calculation area includes three parts: the water pump outlet pipe, the two-stage flap valves and the culvert, as shown in figure 1 .

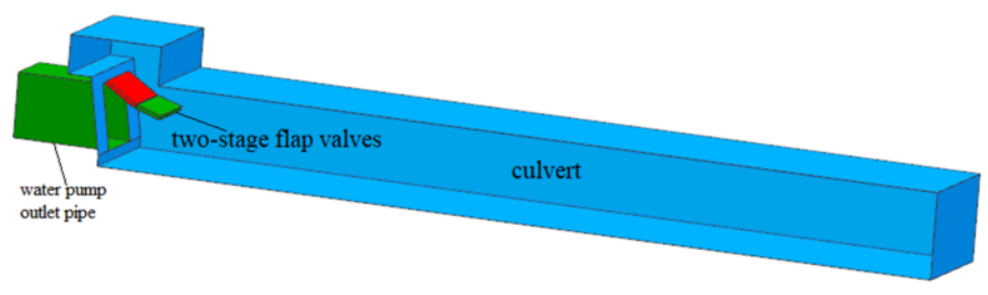

Figure 1. Schematic diagram of calculation area.

\subsection{Numbering}

The structured grid is used to mesh the model [8]. The maximum size of the whole structure grid is 0.2 , and the maximum size of the flap valve panel is 0.05 . Through the 
analysis of grid independence, the total number of grid partition is about 900000 , as shown in figure 2.

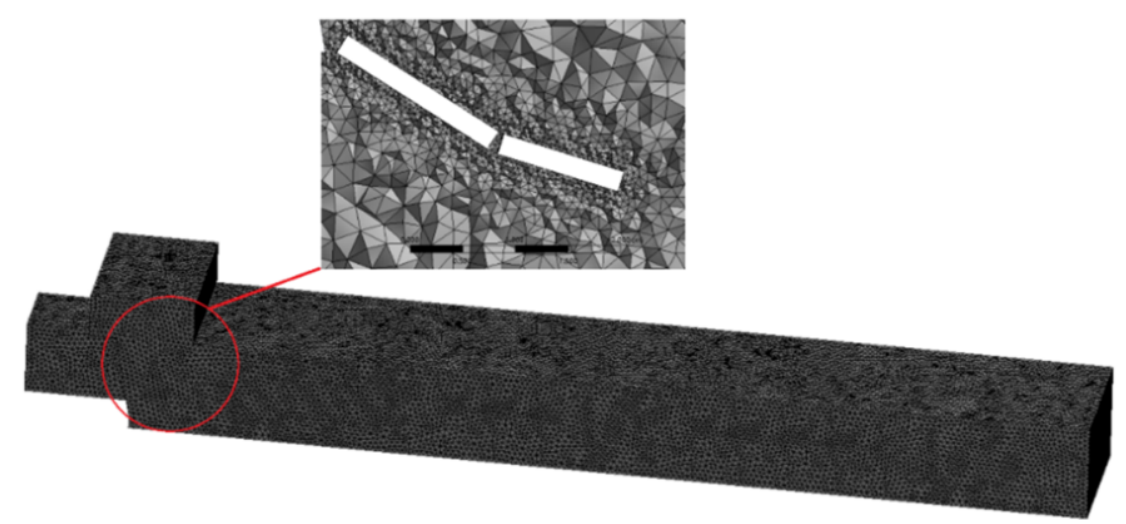

Figure 2. Meshing of computing area.

\subsection{Basic Settings of Numerical Calculation}

ANSYS fluent is used to calculate the calculation area. Velocity inlet condition is adopted for the inlet section [9], and the water velocity is $1.778 \mathrm{~m} / \mathrm{s}$. The outlet section of the culvert is set as the outlet flow, and the relative pressure is a standard atmospheric pressure. The rest of the surfaces are set as wall, and the flap valve is also regarded as the solid wall surface, but it is set as a structural surface separately. The governing equations include the continuity equation of incompressible fluid and the Reynolds time average Navier-Stokes equation [10]. The realizable k-e model is used for simulation, and the separated semi implicit pressure coupling algorithm is used for solution.

\subsection{Numerical Simulation of Hydraulic Loss and Result Analysis}

Based on the research results of the hydraulic loss of the flap valve during opening, numerical simulation adopts the method of controlling the angle of the flap valve to design 4 schemes for comparison. Study the variation of hydraulic loss with flow rate under different opening angles of the flap valve, and the influence on the efficiency of the pump device. The fixed opening angle of the upper and lower beat gate of the design scheme is shown in table 3 .

Table 3. The angle of upper and lower sections of each scheme.

\begin{tabular}{lll}
\hline Programme & Angle of upper gate $\left(^{\circ}\right)$ & Angle of lower gate $\left(^{\circ}\right)$ \\
\hline Scheme 1 & 22 & 37 \\
Scheme 2 & 34 & 56 \\
Scheme 3 & 46 & 64 \\
Scheme 4 & 53 & 70 \\
\hline
\end{tabular}

The curve of the hydraulic loss of the flap valve with the discharge is shown in figure 3 . 


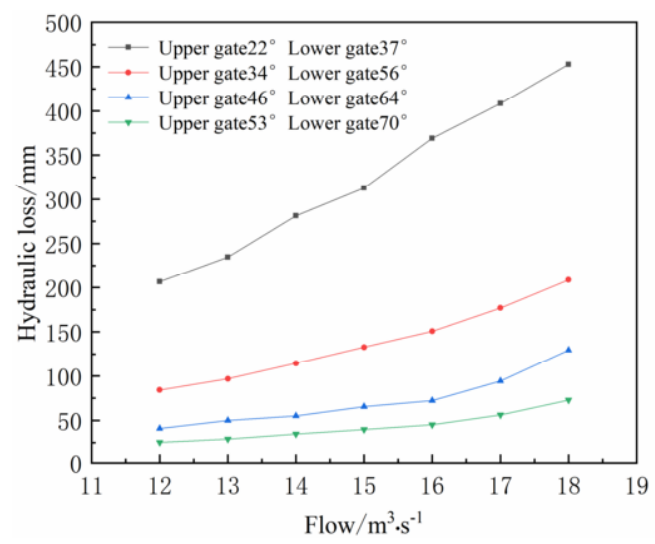

Figure 3. Numerical calculation of hydraulic loss with discharge curve.

At the same opening angle, the hydraulic loss of two-stage flap valves increases with the increase of flow rate. At the same flow rate, the greater the opening angle of the flap valve, the larger the flow area of the cross-section, and the smaller the hydraulic loss of the two-stage flap valves. Compared with the results of the integral flap valve model test in Ref. [4], the two laws are similar. Moreover, the two-stage flap valves have smaller hydraulic loss and larger opening angle, so the hydraulic characteristics of the two-stage flap valves are better. Compared with the data of each scheme, the hydraulic loss of the flap valve in scheme 1 increases rapidly with the increase of flow rate, and the hydraulic loss of the flap valve can reach $375 \mathrm{~mm}$ under the design condition (under the design condition, the flow rate is $16 \mathrm{~m}^{3} / \mathrm{s}$ ). Compared with scheme 1, the increase trend of hydraulic loss in scheme 2 is gradually slowed down, and the hydraulic loss of flap valve is still $151 \mathrm{~mm}$ under the design condition. The hydraulic loss of scheme 3 and scheme 4 in the flow range of $12 \mathrm{~m}^{3} / \mathrm{s} \sim 16 \mathrm{~m}^{3} / \mathrm{s}$ almost does not change with the change of flow, and the hydraulic loss of flap valve is less than $70 \mathrm{~mm}$.

\section{Model Validation}

\subsection{Introduction of Hydraulic Loss Model Test}

The hydraulic loss model test of two-stage flap valves is carried out on the high precision pumping station test bed of Yangzhou University. The test device includes water supply and return equipment and system, flow stabilizing facilities, regulation and control facilities, measuring equipment and facilities, two-stage flap valves model, etc. The model of flap valve is made of transparent plastic. The weight and buoyancy of the flap valve and the corresponding moment are simulated by counterweight method. The shape of the prototype and model flap valve is similar. The model test device is shown in figure 4. 


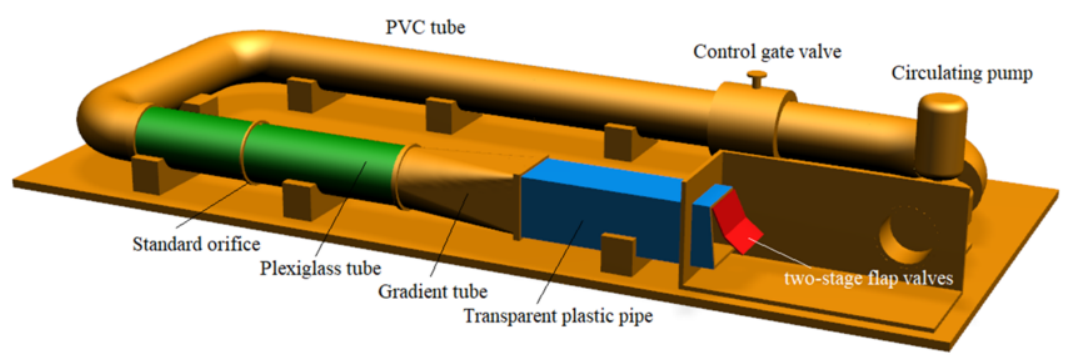

Figure 4. Schematic diagram of model test device.

The scale of prototype and model is 5:1, and the water level inside and outside of the flap valve is displayed by microcomputer data acquisition system. The water level measuring point outside the gate is located under the gate, and the water level measuring point inside the gate is located at the bottom of the pipe $50 \mathrm{~cm}$ inside the gate. The flow is measured with standard orifice. The standard orifice plate is made of stainless steel, and the pressure is taken by flange. The velocity measuring points are set at $0.7 \mathrm{~m}$ inside and $1.5 \mathrm{~m}$ outside the outlet of the flap valve model, and the loss of the pipeline outlet with two-stage flap valves are taken as the local hydraulic loss of the flap valve. Bernoulli equation is used to calculate the hydraulic loss of the two-stage flap valves:

$$
\Delta h_{p}=Z_{1}-Z_{2}+\frac{P_{1}-P_{2}}{\rho g}+\frac{\varsigma_{1} v_{1}^{2}-\varsigma_{2} v_{2}^{2}}{2 g}-\xi_{1} \frac{v_{1}^{2}-v_{2}^{2}}{2 g}
$$

where: $Z_{1}, Z_{2}$ is the potential energy of the same reference plane; $P_{1}, P_{2}$ is the center pressure of the channel before and after the outlet section of the channel; $v_{1}, v_{2}$ is the average velocity before and after the outlet section; $\varsigma_{1}, \varsigma_{2}$ is the kinetic energy correction coefficient; $\xi_{1}$ is the local loss coefficient of outlet section expansion.

\subsection{Analysis of Hydraulic Loss Model Test Results}

For the convenience of reference and comparison, the scheme design of physical model test is consistent with that of numerical simulation. The results of model test are shown in figure 5.

Compared with the data obtained from numerical simulation, the law of hydraulic loss and flow change in model test is consistent with that of numerical simulation. Moreover, the hydraulic loss value of the flap valve obtained by the numerical simulation is close to that obtained by the model test, which indicates that the numerical simulation has a certain degree of credibility.

Under the design condition (the flow rate is $16 \mathrm{~m}^{3} / \mathrm{s}$ ), the hydraulic loss of the flap valve and the efficiency decrease of the pump device are compared between the numerical calculation and the model test. The results are shown in table 4.

It can be seen from the data in the table that when the opening angle of the twostage flap valves are small, the flap valve has a greater impact on the efficiency of the pump device. When the opening of the upper gate reaches $46^{\circ}$ and the opening of the lower gate reaches $64^{\circ}$, the efficiency reduction caused by the hydraulic loss of the flap valve in the numerical simulation and model test has been reduced to about $3 \%$. With 
the increase of the opening angle, the impact of the flap valve on the pump performance and the overall project operation is gradually weakening. This law is consistent with the law of efficiency decrease of integral flap valve in Ref. [2]. It shows that the hydraulic loss of the two-stage flap valves tends to be stable when the opening angle of the upper gate is greater than $46^{\circ}$ and the opening angle of the lower gate is greater than $64^{\circ}$. The influence on the performance of the pump device is gradually weakened, which can meet the requirements of the economic and safe operation of the pump station.

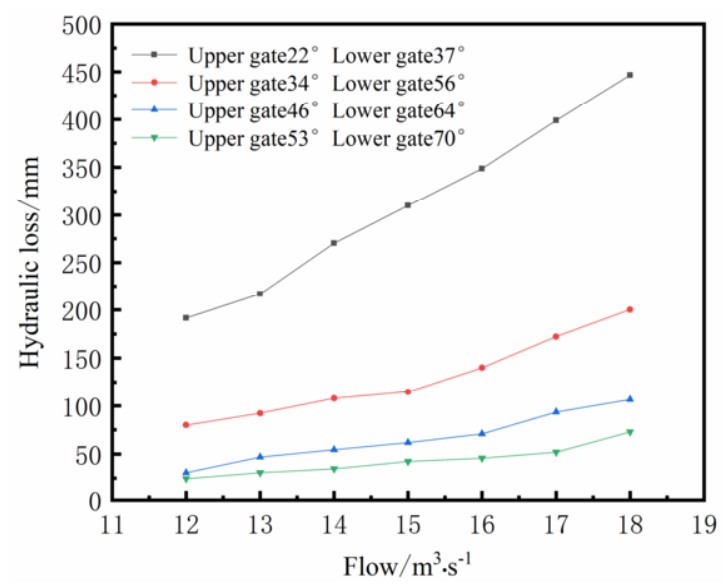

Figure 5. Curve of hydraulic loss with discharge in model test.

Table 4. Comparison of numerical calculation and model test results.

\begin{tabular}{lllll}
\hline \multirow{2}{*}{ Programme } & \multicolumn{2}{l}{ Numerical calculation } & \multicolumn{2}{l}{ Model test } \\
\cline { 2 - 5 } & Hydraulic loss (m) & Efficiency decline (\%) & Hydraulic loss (m) & Efficiency decline (\%) \\
\hline Scheme 1 & 0.369 & 16.18 & 0.348 & 15.26 \\
Scheme 2 & 0.151 & 6.62 & 0.140 & 6.14 \\
Scheme 3 & 0.072 & 3.16 & 0.069 & 3.03 \\
Scheme 4 & 0.045 & 1.97 & 0.043 & 1.89 \\
\hline
\end{tabular}

\subsection{Discussion on the Relationship between Hydraulic Loss and Flow}

The relationship between the hydraulic loss and the flow of the flap valve is obtained by fitting the hydraulic loss and the flow of the flap valve under four different opening angle operation schemes of the model test. The reasonable degree of curve fitting is determined by $\mathrm{R}^{2}$ value (The closer the $\mathrm{R}^{2}$ value is to 1 , the more reasonable the curve fitting is). The fitting curves of the four schemes are shown in figure 6 . The fitting curve of hydraulic loss and discharge is shown in table 5.

By fitting the hydraulic loss and flow of the two-stage flap valves, it is found that the relationship between hydraulic loss and flow is cubic under the condition of opening angle of the two-stage flap valves in scheme 1; In scheme 2, the relationship between hydraulic loss and flow is quartic; The relationship between hydraulic loss and flow rate is quintic under the condition of opening angle of two-stage flap valves in scheme 3 and scheme 4. Compared with the research results of Ref. [2], the relationship between hydraulic loss and flow of two-stage flap valves do not meet the 
relationship of $\Delta \mathrm{h}=\mathrm{SQ}^{2}$, just like that of integral flap valve. Moreover, with the increase of the opening angle of the two-stage flap valves, the order of the relationship between the hydraulic loss and the flow increases. Because the flow from the outlet of the pump has a certain circulation, it has a certain influence on the flow pattern in the outlet channel. It will form a certain bias phenomenon, which will have a certain impact on the hydraulic loss of the flap valve.

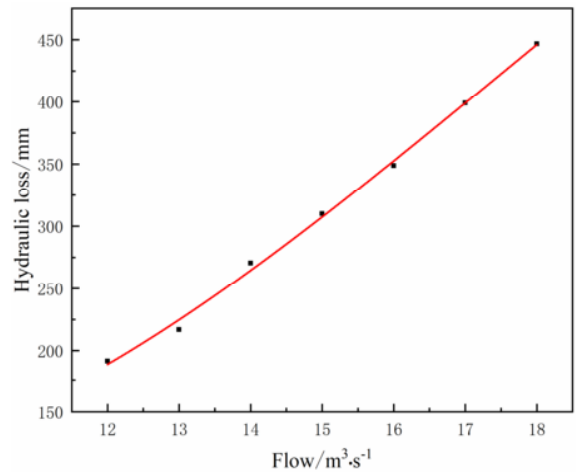

(a) Scheme 1

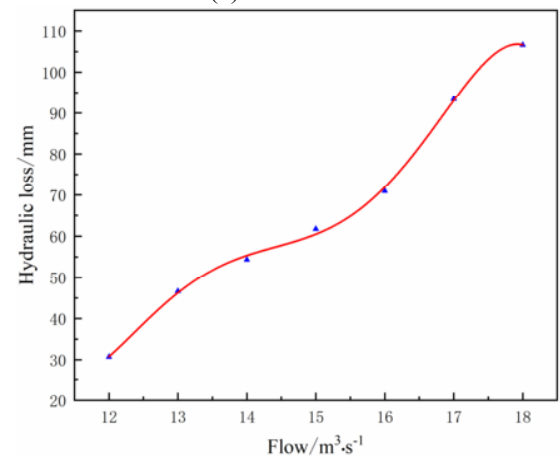

(c) Scheme 3

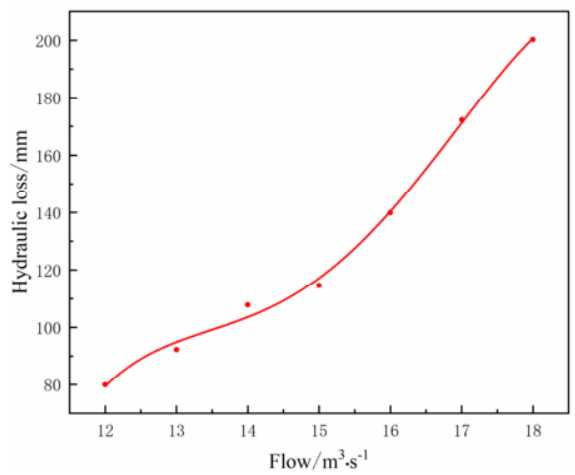

(b) Scheme 2

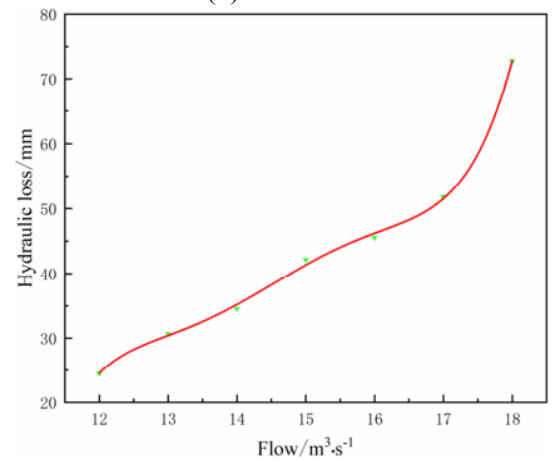

(d) Scheme 4

Figure 6. Fitting curve of relationship between hydraulic loss and discharge of each scheme.

Table 5. Relationship between hydraulic loss and flow fitting curve.

\begin{tabular}{llr}
\hline ProgrammeFitting curve relation & $\mathbf{R}^{2}$ value \\
\hline Scheme 1 & $\Delta h=-0.153 Q^{3}+8.001 Q^{2}-92.727 Q+413.61$ & 0.99516 \\
Scheme 2 & $\Delta h=-0.285 Q^{4}+17.356 Q^{3}-390.49 Q^{2}+3871.8 Q-14222$ & 0.99134 \\
Scheme 3 & $\Delta h=-0.11929 Q^{5}+8.6728 Q^{4}-250.22 Q^{3}+3581.1 Q^{2}-25414 Q+71551$ & 0.99466 \\
Scheme 4 & $\Delta h=0.07871 Q^{5}-5.7209 Q^{4}+165.69 Q^{3}-2390.1 Q^{2}+17176 Q-491900.99318$ \\
\hline
\end{tabular}

\section{Conclusion}

(a) At the same opening angle, the hydraulic loss of two-stage flap valves increases with the increase of flow; Under the same flow condition, the larger the opening angle of the flap valve is, the larger the cross-section flow area is, and the smaller the hydraulic loss is. 
(b) The two-stage flap valves have better structure and hydraulic characteristics. When the opening angle of the upper gate is greater than $46^{\circ}$ and the opening angle of the lower gate is greater than $64^{\circ}$, the hydraulic loss of the two-stage flap valves is less than $70 \mathrm{~mm}$ and tends to be stable. And the impact on the performance of the pump device is gradually weakened, which can meet the requirements of economic and safe operation of the pump station.

(c) The relationship between the hydraulic loss and the flow of the two-stage flap valves are no longer quadratic. With the increase of the opening angle of the two-stage flap valves, the order of the relationship between hydraulic loss and flow increases. This may be related to the flow pattern of the outlet channel of the pump. In the future, we will focus on the relationship between the flow pattern of the outlet channel of the pump and the hydraulic loss of the flap valve.

\section{References}

[1] Zhu H G and Fen H M 1996 Model test research on hydraulic head loss coefficient of double-stage flap valve Journal of Hydraulic Engineering 09 63-67.

[2] Yang F, Zhou J R and Liu C 2011 Numerical simulation and experiment on resistance loss of flap gate Transactions of the Chinese Society for Agricultural Machinery 42 (09) 108-112+44.

[3] Zhu H G and Luo J Q 1995 Effect of pump-gate head loss on pump unit efficiency China Rural Water and Hydropower 04 41-42.

[4] He C L, You C T and Dong S 2002 Experimental study on flap valve of pump model device Design of Water Resources \& Hydroelectric Engineering 21 (04) 45-47.

[5] Chu X 1985 Development and application of double-stage flap valve Drainage and Irrigation Machinery 06 5-8.

[6] Chu X and Feng H M 1982 Discussion on the theory of double-stage flap valve Journal of Jiangsu Agricultural University 04 1-13.

[7] Xie R S, Xiang C, Chen Y Y, Fang H F and Wang L 2012 Analysis of flow capacity of gate with small flap valve base on CFD Water Resources and Power 36 (12) 156-158.

[8] Cloete S, Johansen S T and Amini S 2015 Grid independence behaviour of fluidized bed reactor simulations using the Two Fluid Model: Effect of particle size Powder Technology 269 153-165.

[9] Xu L, Lu W G, Lu L G, Dong L and Wang Z F 2014 Flow patterns and boundary conditions for inlet and outlet conduits of large pump system with low head Applied Mathematics and Mechanics (English Edition) 35 (6) 675-688.

[10] Jafarzadeh B, Hajari A, Alishahi M M and Akbari M H 2011 The flow simulation of a low-specificspeed high-speed centrifugal pump Applied Mathematical Modelling 35 (1) 242-249. 